\title{
Bivariate gamma type distributions for modeling wireless performance metrics
}

\author{
Andriette Bekker, Johan Ferreira * \\ Department of Statistics, University of Pretoria, South Africa.
}

\begin{abstract}
In this paper a bivariate gamma type distribution, its noncentral counterpart, and a linked bivariate Weibullised gamma type distribution, following an elliptical assumption, are proposed and studied. The adaptability of this contribution is illustrated with the outage probability performance metric, where the proposed bivariate gamma type distributions may act as alternatives to existing fading models in wireless communications.
\end{abstract}

Keywords Elliptical, Fading, MIMO system, Noncentral, Outage probability, Weibullised

AMS 2010 subject classifications 60E05; 62E15; 62H10, 94A99.

DOI: $10.19139 /$ soic.v6i3.575

\section{Introduction}

Bivariate probability distributions have received significant attention in literature (see [1]), and even more so within the context of wireless systems (see [14], [18], [17], [5], [25], [6], [22], [11], [2]). Bivariate gamma distributions are used to describe the fading of signal channels between either two transmitters or two receivers - specifically in the modeling of dual-antenna wireless systems operating over correlated branches. Bivariate gamma distributions in particular have been of interest due to its pliable- and computable mathematical nature, exhibiting satisfactory fits to measured data subjected to multipath/shadowing fading (see [17], [20]). This paper proposes a new class of (correlated) bivariate gamma distributions emanating from the elliptical arena, thereby creating versatile bivariate distributions. These bivariate gamma type distributions (called as such due to the elliptical origin, justifying the word "type") are studied via their probability density function (pdf), cumulative distribution function (cdf), and product moment. The bivariate Nakagami distribution is also meaningful within the wireless communications framework as it models the envelope, or amplitude, of a received wireless signal. It is obtained as a transformation from a bivariate gamma distributed variable; thus, to this effect, a bivariate Weibullised gamma type distribution is also proposed with a bivariate Nakagami type distribution as special case. Via these contributions we don't only gain valuable insight into the distributional structure of these distributions, but also expand the knowledge base of alternative candidates for modeling within the wireless communications domain; and the results in this paper may be used for analyzing dual-antenna systems.

The mathematical representation of the distributions in this paper, which originates from the elliptical platform, is noteworthy (see [2]). The representation allows for flexible and alternative choices of underlying process distributions for the practitioner; usually chosen according to measurements taken in practice (such as power of wireless signals). This paper investigates the usual underlying normal assumption, as well as an underlying $t$ assumption; due to the well-known statistical relevance of these two candidates. [5] discussed some early thoughts

${ }^{*}$ Correspondence to: Johan Ferreira (Email: johan.ferreira@up.ac.za). Department of Statistics, University of Pretoria. Pretoria, South Africa (0002).

ISSN 2310-5070 (online) ISSN 2311-004X (print)

Copyright (C) 2018 International Academic Press 
regarding the assumption of the underlying normality of fading channels, noting that Rayleigh fading stems from normal random processes. However, this normality is an approximation and the restriction thereto is questionable. In fact, [3] proposes and implements the complex matrix variate $t$ model as underlying process for a wireless communications system and motivates its importance within the field - therefore a more general assumption rather than normal may not be that far from reality (also see [15]).

The models which describe fading of signals are inherently statistical distributions in nature, where the behaviour of signals and their degradation can be described by the Rician- and Rayleigh distributions respectively. Rician fading is of interest when there is a direct line-of-sight (LOS) component between transmitters and receivers; mathematically this translates to assuming a nonzero mean for the underlying process; with Rayleigh fading corresponding to a zero mean for the underlying process (see [9] and [10]). [13] derives the distribution of $R=\sqrt{X^{2}+Y^{2}}$ where $X, Y$ are independent normally distributed random variables with mean $\mu_{1}$ and $\mu_{2}$ respectively and common covariance $\sigma^{2}$; this $R$ represents Rician fading, and models the envelope of the process stemming from $X$ and $Y$. By substituting the underlying normal assumption to that of elliptical implies that both Rician- and Rayleigh distributions have to be reconsidered from the elliptical viewpoint as well. The univariate elliptical distribution acts as impetus of construction of the fading distributions, and eventually the bivariate type distributions in this paper. The result here is analogous to the main result from [4]. If $X$ follows an elliptical distribution with mean $\mu$, variance $\sigma^{2}$, generator function $h$, and has pdf:

$$
f(x)=\frac{1}{\sigma} h\left(-\frac{(x-\mu)^{2}}{2 \sigma^{2}}\right)
$$

then there exists a scalar weight function $\mathcal{W}(\cdot)$ on $\mathbb{R}^{+}$such that:

$$
\begin{aligned}
f(x) & =\int_{\mathbb{R}^{+}} \frac{1}{\sqrt{2 \pi} \sigma t^{-\frac{1}{2}}} \exp \left(-\frac{(x-\mu)^{2}}{2 \sigma^{2} t^{-1}}\right) \mathcal{W}(t) d t \\
& =\int_{\mathbb{R}^{+}} f_{N\left(\mu, \sigma^{2} t^{-1}\right)}(x \mid t) \mathcal{W}(t) d t
\end{aligned}
$$

where $f_{N\left(\mu, \sigma^{2} t^{-1}\right)}(x \mid t)$ is the pdf of a normal distribution with mean $\mu$ and variance $\sigma^{2} t^{-1}$. Two well known members of the elliptical distribution is the normal distribution with weight:

$$
\mathcal{W}(t)=\delta(t-1)
$$

known as the dirac delta function (with property $\int_{0}^{\infty} f(x) \delta(x) d x=f(0)$ ); and the $t$ distribution with weight:

$$
\mathcal{W}(t)=\frac{\left(\frac{v}{2}\right)^{\frac{v}{2}}}{\Gamma\left(\frac{v}{2}\right)} t^{\frac{v}{2}-1} \exp \left(-\frac{v t}{2}\right)
$$

with $v>0$ degrees of freedom (see [4]) and where $\Gamma(\cdot)$ denotes the gamma function.

Following the representation in (1), the pdf of $R$, where $X$ and $Y$ are independent elliptically distributed random variables, with mean $\mu_{1}$ and $\mu_{2}$, and common covariance $\sigma^{2} t^{-1}$ is given by:

$$
f(r)=\int_{\mathbb{R}^{+}} \frac{r}{\sigma^{2} t^{-1}} \exp \left(-\frac{r^{2}+s^{2}}{2 \sigma^{2} t^{-1}}\right) I_{0}\left(\frac{r s}{\sigma^{2} t^{-1}}\right) \mathcal{W}(t) d t
$$

where $r>0, s^{2}=\mu_{1}^{2}+\mu_{2}^{2}$, and $I_{0}(\cdot)$ is the $0^{t h}$ order Bessel function of the $1^{\text {st }}$ kind. This distribution is referred to as a Rician type distribution. By choosing $\mathcal{W}(t)$ as (2), (4) simplifies to

$$
f(r)=\frac{r}{\sigma^{2}} \exp \left(-\frac{r^{2}+s^{2}}{2 \sigma^{2}}\right) I_{0}\left(\frac{r s}{\sigma^{2}}\right)
$$


for $r>0$, known as the Rician distribution (see [20], pp. 201, and [13]). When $\mu_{1}=\mu_{2}=0$, (4) simplifies to:

$$
f(r)=\int_{\mathbb{R}^{+}} \frac{r}{\sigma^{2} t^{-1}} \exp \left(-\frac{r^{2}}{2 \sigma^{2} t^{-1}}\right) \mathcal{W}(t) d t,
$$

and referred to as a Rayleigh type distribution. This is used as a departure point from where we systematically construct bivariate gamma type distributions with their origins following this elliptical assumption.

This paper's contribution can be summarised as follows:

1. A bivariate gamma type distribution is developed and particular characteristics originating from the elliptical distribution, with the work of [18] as a special case;

2. A noncentral counterpart of this bivariate gamma type distribution inspired by the methodology of [7] is proposed;

3. A bivariate Weibullised gamma type distribution and particular characteristics are developed, with the work of [17] as a special case; and

4. Speculative comparison of the new models in terms of the performance metric, outage probability.

The paper is laid out in the following way. To set the platform, Section 2 presents the description of methodology and construction of univariate gamma type distributions from the elliptical distribution. Section 3 proposes a bivariate gamma type distribution with some statistical characteristics as well as a bivariate noncentral gamma type distribution. Section 4 pays attention to the bivariate Weibullised gamma type that follows naturally from the proposed bivariate gamma type, with a bivariate Nakagami type distribution as proposed alternative model for the envelope. Section 5 discusses a possible application in terms of outage probability of a wireless fading channel subject to the developed models, and final thoughts are contained in the Section 6. The Appendix contains simplified results of interest to this paper.

\section{Methodology and univariate construction}

Suppose that $X$ follows a Rayleigh type distribution as in (6). The transformation $Y=\frac{X^{2} \Omega}{2 \sigma^{2}}$ with $\frac{d x}{d y}=\sigma \sqrt{\frac{2}{\Omega}} \frac{1}{2} y^{-\frac{1}{2}}$ results in $Y$ following an exponential type distribution with parameter $\Omega>0$ and pdf:

$$
\begin{aligned}
f(y) & =\int_{0}^{\infty}\left(\frac{1}{\Omega t^{-1}} \exp \left(-\frac{1}{\Omega t^{-1}} y\right)\right) \mathcal{W}(t) d t \\
& =\int_{0}^{\infty} f(y \mid t) \mathcal{W}(t) d t
\end{aligned}
$$

with $y>0$, where $f(y \mid t)$ is the pdf of an exponential distribution with parameter $\Omega t^{-1}>0$ i.e. $Y \sim$ $\operatorname{Exp}(\Omega, \mathcal{W}(\cdot))$. This pdf in (7) forms the basic element of the composition of the proposed bivariate distributions in this paper. Using [8], p. 346, eq. 3.381.4, the Laplace transform of $Y$ is given by:

$$
\begin{aligned}
\mathcal{L}_{Y}(s) & =E(\exp (-s Y)) \\
& =\frac{1}{1+\Omega t^{-1} s} .
\end{aligned}
$$

The Laplace transform of the variables is a vital component to derive the pdf of the bivariate gamma type distribution in subsequent sections. 
Suppose we have i.i.d. random variables $Y_{1}\left|t, Y_{2}\right| t, \ldots, Y_{m} \mid t$ which is distributed as $\operatorname{Exp}\left(\Omega t^{-1}\right)$. Then $U=$ $\sum_{i=1}^{m} Y_{i} \mid t$ is distributed as Gamma $\left(\Omega t^{-1}, m\right)$. Ergo, if we have i.i.d. random variables $Y_{1}, Y_{2}, \ldots, Y_{m}$ which is distributed as $\operatorname{Exp}(\Omega, \mathcal{W}(\cdot))$, then $U=\sum_{i=1}^{m} Y_{i}$ is distributed as a gamma type distribution, denoted as $\operatorname{Gamma}(\Omega, m, \mathcal{W}(\cdot))$, with Laplace transform

$$
\begin{aligned}
\mathcal{L}_{U}(s) & =\int_{0}^{\infty}\left(1+\Omega t^{-1} s\right)^{-m} \mathcal{W}(t) d t \\
& =\int_{0}^{\infty} \mathcal{L}_{U}(s \mid t) \mathcal{W}(t) d t .
\end{aligned}
$$

The pdf of this gamma type distribution follows via an inverse Laplace transform as:

$$
\begin{aligned}
f(u) & =\int_{0}^{\infty}\left(\frac{1}{\left(\Omega t^{-1}\right)^{m} \Gamma(m)} u^{m-1} \exp \left(-\frac{1}{\Omega t^{-1}} u\right)\right) \mathcal{W}(t) d t \\
& =\int_{0}^{\infty} f(u \mid t) \mathcal{W}(t) d t
\end{aligned}
$$

for $u>0$ where $m, \Omega>0$. For modelling the envelope, the variable $W=\left(\frac{U}{m}\right)^{\frac{1}{\beta}}, \beta>0$ is of interest, with pdf

$$
f(w)=\frac{\beta m^{m}}{\Omega^{m} \Gamma(m)} w^{\beta m-1} \int_{0}^{\infty} t^{m} \exp \left(-\frac{m}{\Omega t^{-1}} w^{\beta}\right) \mathcal{W}(t) d t
$$

which is referred to as a Weibullised gamma type distribution with the Nakagami type distribution as a special case when $\beta=2$.

\section{Bivariate gamma type distribution and characteristics}

The construction of the bivariate gamma type distribution is now described. Following a similar approach as in [18], suppose

$$
\begin{aligned}
U_{1} & =\sum_{k=1}^{m_{1}} Y_{k} \\
U_{2} & =U_{2 a}+U_{2 b}=\sum_{h=1}^{m_{1}} Y_{h}+\sum_{h=m_{1}+1}^{m_{2}} Y_{h}
\end{aligned}
$$

where $Y_{k}\left(k=1, \ldots, m_{1}\right)$ and $Y_{h}\left(h=1, \ldots, m_{2}\right)\left(m_{2} \geq m_{1}\right)$ are independent exponential type random variables. Thus,

$$
\begin{array}{lll}
Y_{k} \sim \operatorname{Exp}\left(\Omega_{1}, \mathcal{W}(\cdot)\right) & \text { with } Y_{k} \mid t \sim \operatorname{Exp}\left(\Omega_{1}\right) ; \\
Y_{h} \sim \operatorname{Exp}\left(\Omega_{2}, \mathcal{W}(\cdot)\right) & \text { with } Y_{h} \mid t \sim \operatorname{Exp}\left(\Omega_{2}\right) ; \\
U_{1} \sim \operatorname{Gamma}\left(\Omega_{1}, m_{1}, \mathcal{W}(\cdot)\right) & \text { with } U_{1} \mid t \sim \operatorname{Gamma}\left(\Omega_{1}, m_{1}\right) ; \text { and } \\
U_{2} \sim \operatorname{Gamma}\left(\Omega_{2}, m_{2}, \mathcal{W}(\cdot)\right) & \text { with } U_{2} \mid t \sim \operatorname{Gamma}\left(\Omega_{2}, m_{2}\right) .
\end{array}
$$


We are interested in the bivariate distribution of $U_{1}$ and $U_{2}$, with Laplace transform of $\left(U_{1}, U_{2}\right)$ given as

$$
\mathcal{L}_{U_{1}, U_{2}}\left(s_{1}, s_{2}\right)=\int_{0}^{\infty} \mathcal{L}_{U_{1}, U_{2}}\left(s_{1}, s_{2} \mid t\right) \mathcal{W}(t) d t .
$$

Note that the Laplace transform of $\left(U_{1}, U_{2} \mid t\right)$ can be decomposed as:

$$
\begin{aligned}
\mathcal{L}_{U_{1}, U_{2}}\left(s_{1}, s_{2} \mid t\right) & =E\left(\exp \left(-s_{1} U_{1}-s_{2} U_{2 a}\right) \exp \left(-s_{2} U_{2 b}\right)\right) \\
& =\mathcal{L}_{U_{1}, U_{2 a}}\left(s_{1}, s_{2} \mid t\right) \mathcal{L}_{U_{2 b}}\left(s_{2} \mid t\right)
\end{aligned}
$$

since $U_{1}$ and $U_{2 a}$ is independent of $U_{2 b}$.

From (8) and [14] it follows that

$$
\mathcal{L}_{U_{2 b}}\left(s_{2} \mid t\right)=\frac{1}{\left(\Omega_{2} t^{-1}\right)^{m_{2}-m_{1}}\left(s_{2}+\frac{1}{\Omega_{2} t^{-1}}\right)^{m_{2}-m_{1}}},
$$

also, the Laplace transform of $\left(U_{1}, U_{2 a} \mid t\right)$ is

$$
\begin{aligned}
& \mathcal{L}_{U_{1}, U_{2 a}}\left(s_{1}, s_{2} \mid t\right) \\
= & \left(\Omega_{1} t^{-1}\right)^{-m_{1}}\left(\Omega_{2} t^{-1}\right)^{-m_{1}}(1-\rho)^{-m_{1}} \\
& \times\left[\left(s_{1}+\frac{1}{\left(\Omega_{1} t^{-1}\right)(1-\rho)}\right)\left(s_{2}+\frac{1}{\left(\Omega_{2} t^{-1}\right)(1-\rho)}\right)-\frac{\rho}{\left(\Omega_{1} t^{-1}\right)\left(\Omega_{2} t^{-1}\right)\left(1-\rho^{2}\right)}\right]^{-m_{1}}
\end{aligned}
$$

where $\rho$ represents the correlation coefficient defined by $\rho_{U_{1}, U_{2 a}}=\frac{E\left(U_{1} U_{2 a}\right)-E\left(U_{1}\right) E\left(U_{2 a}\right)}{\sqrt{E\left(U_{1}^{2}\right)-E\left(U_{1}\right)^{2}} \sqrt{E\left(U_{2 a}^{2}\right)-E\left(U_{2 a}\right)^{2}}}$. Combining (13) and (14) leaves

$$
\begin{aligned}
& \mathcal{L}_{U_{1}, U_{2}}\left(s_{1}, s_{2} \mid t\right) \\
= & \left(\Omega_{1} t^{-1}\right)^{-m_{1}}\left(\Omega_{2} t^{-1}\right)^{-m_{2}}(1-\rho)^{-m_{1}}\left[s_{2}+\frac{1}{\Omega_{2} t^{-1}}\right]^{-\left(m_{2}-m_{1}\right)} \\
& \times\left[\left(s_{1}+\frac{1}{\left(\Omega_{1} t^{-1}\right)(1-\rho)}\right)\left(s_{2}+\frac{1}{\left(\Omega_{2} t^{-1}\right)(1-\rho)}\right)-\frac{\rho}{\left(\Omega_{1} t^{-1}\right)\left(\Omega_{2} t^{-1}\right)\left(1-\rho^{2}\right)}\right]^{-m_{1}} .
\end{aligned}
$$

Substituting (15) in (11) gives

$$
\begin{aligned}
& \mathcal{L}_{U_{1}, U_{2}}\left(s_{1}, s_{2}\right) \\
= & \int_{0}^{\infty}\left(\Omega_{1} t^{-1}\right)^{-m_{1}}\left(\Omega_{2} t^{-1}\right)^{-m_{2}}(1-\rho)^{-m_{1}}\left[s_{2}+\frac{1}{\Omega_{2} t^{-1}}\right]^{-\left(m_{2}-m_{1}\right)} \\
& \times\left[\left(s_{1}+\frac{1}{\left(\Omega_{1} t^{-1}\right)(1-\rho)}\right)\left(s_{2}+\frac{1}{\left(\Omega_{2} t^{-1}\right)(1-\rho)}\right)-\frac{\rho}{\left(\Omega_{1} t^{-1}\right)\left(\Omega_{2} t^{-1}\right)\left(1-\rho^{2}\right)}\right]^{-m_{1}} \mathcal{W}(t) d t .
\end{aligned}
$$

Applying a binomial expansion ([8], p. 25, eq. 1.111) of (15) leaves the Laplace transform in series form as

$$
\begin{aligned}
& \mathcal{L}_{U_{1}, U_{2}}\left(s_{1}, s_{2} \mid t\right) \\
= & \left(\Omega_{1} t^{-1}\right)^{-m_{1}}\left(\Omega_{2} t^{-1}\right)^{-m_{2}}(1-\rho)^{-m_{1}}\left[s_{2}+\frac{1}{\Omega_{2} t^{-1}}\right]^{-\left(m_{2}-m_{1}\right)} \\
& \times\left[\left(s_{1}+\frac{1}{\left(\Omega_{1} t^{-1}\right)(1-\rho)}\right)\left(s_{2}+\frac{1}{\left(\Omega_{2} t^{-1}\right)(1-\rho)}\right)\right]^{-m_{1}} \\
& \times \sum_{k=0}^{\infty} \frac{\left(m_{1}\right)_{k}}{k !}\left(\frac{\rho}{\left(\Omega_{1} t^{-1}\right)\left(\Omega_{2} t^{-1}\right)\left(1-\rho^{2}\right)}\right)^{k} \frac{1}{\left[\left(s_{1}+\frac{1}{\left(\Omega_{1} t^{-1}\right)(1-\rho)}\right)\left(s_{2}+\frac{1}{\left(\Omega_{2} t^{-1}\right)(1-\rho)}\right)\right]^{k}}
\end{aligned}
$$


Substituting (17) into (15), and subsequently (15) into (16), and then applying an inverse Laplace transform on (16), the pdf of the bivariate gamma type distribution is obtained as

$$
\begin{aligned}
f\left(u_{1}, u_{2}\right)= & (1-\rho)^{m_{2}} \sum_{k=0}^{\infty} \frac{\left(m_{1}\right)_{k}}{k !} \rho^{k}\left(\frac{1}{\Omega_{1}(1-\rho)}\right)^{m_{1}+k}\left(\frac{1}{\Omega_{2}(1-\rho)}\right)^{m_{2}+k} \\
& \times \frac{u_{1}^{m_{1}+k-1}}{\Gamma\left(m_{1}+k\right)} \frac{u_{2}^{m_{2}+k-1}}{\Gamma\left(m_{2}+k\right)} \int_{0}^{\infty} \exp \left[-t\left(\frac{u_{1}}{\Omega_{1}(1-\rho)}+\frac{u_{2}}{\Omega_{2}(1-\rho)}\right)\right] \\
& \times{ }_{1} F_{1}\left(m_{2}-m_{1}, m_{2}+k ; \frac{t \rho}{\Omega_{2}(1-\rho)} u_{2}\right) t^{m_{1}+m_{2}+2 k} \mathcal{W}(t) d t
\end{aligned}
$$

for $u_{1}, u_{2}>0$, where $m_{1}, m_{2}, \Omega_{1}, \Omega_{2}>0, \quad-1<\rho<1$, and $m_{2} \geq m_{1} .{ }_{1} F_{1}(\cdot)$ denotes the confluent hypergeometric function.

For weights (2) and (3) respectively, the results are given in Appendix equations (34) and (35). The contourplots of these specific cases are illustrated for $m_{1}=10, m_{2}=12, \Omega_{1}=2.5, \Omega_{2}=1.5$, and arbitrary choices for other parameters.
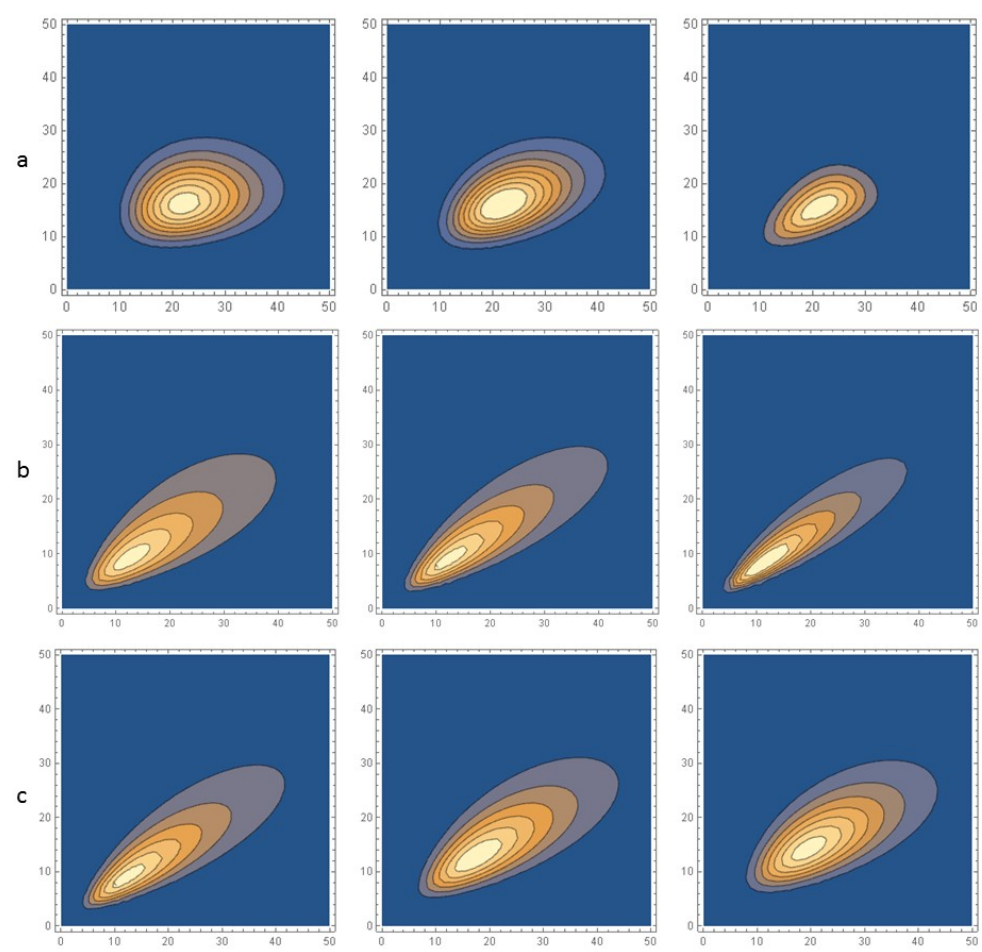

\section{Figure 1}
(a) Pdf (34) for varying $\rho=0.3,0.5,0.7$ (fltr).
(b) Pdf (35) for $v=5$ and varying $\rho=0.3,0.5,0.7$ (fltr).
(c) Pdf (35) for $\rho=0.5$ and varying $v=5,15,30$ (fltr).

In Figure 1 the effect of increasing $\rho$ on the pdfs are observable ((a) and (b)), particularly indicating increased concentration (or correlation) between the variables. Figure 1 (c) indicate the effect on the tails for increasing values of $v$. 
If $\left(U_{1}, U_{2}\right)$ is distributed with pdf (18), then the marginal pdf of $U_{i}, i=1,2$ is given by:

$$
f\left(u_{i}\right)=\frac{u_{i}^{m_{i}-1}}{\Omega_{i}^{m_{i}} \Gamma\left(m_{i}\right)} \int_{0}^{\infty} t^{m_{i}} \exp \left[-t\left(\frac{u_{i}}{\Omega_{i}}\right)\right] \mathcal{W}(t) d t
$$

where $u_{i}>0$ for $m_{i}, \Omega_{i}>0$.

An expression for the product moment of this bivariate gamma type distribution with pdf (18) is given by:

$$
\begin{aligned}
E\left(U_{1}^{n} U_{2}^{j}\right)= & \Omega_{1}^{n} \Omega_{2}^{j}(1-\rho)^{n+j+m_{2}} \sum_{k=0}^{\infty} \frac{\left(m_{1}\right)_{k}}{k !} \rho^{k} \frac{\Gamma\left(n+m_{1}+k\right) \Gamma\left(j+m_{2}+k\right)}{\Gamma\left(m_{1}+k\right) \Gamma\left(m_{2}+k\right)} \\
& \times{ }_{2} F_{1}\left(m_{2}-m_{1}, j+m_{2}+k ; m_{2}+k ; \rho\right) \int_{0}^{\infty} t^{-(n+j)} \mathcal{W}(t) d t
\end{aligned}
$$

where $n, j>0$, and $m_{2}, m_{1}, \Omega_{1}, \Omega_{2}>0,-1<\rho<1$, and $m_{2} \geq m_{1}$. Simplified results of (19) and (20) for weights (2) and (3) are given in the Appendix as equations (36) through (39).

Using (38) and (39), the Pearson correlation coefficient

$$
\rho_{U_{1}, U_{2}}=\frac{E\left(U_{1} U_{2}\right)-E\left(U_{1}\right) E\left(U_{2}\right)}{\sqrt{E\left(U_{1}^{2}\right)-E\left(U_{1}\right)^{2}} \sqrt{E\left(U_{2}^{2}\right)-E\left(U_{2}\right)^{2}}}
$$

is illustrated for $m_{2}=12, \Omega_{1}=2.5, \Omega_{2}=1.5, \rho=0.5$, and $v=10,30$.

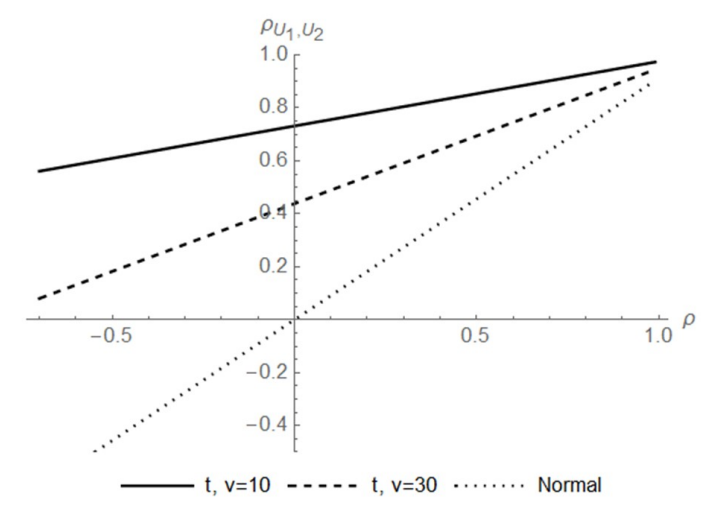

Figure 2 Correlation $\rho_{U_{1}, U_{2}}$ using (38) and (39).

Figure 2 illustrates increased (21) for (39) when compared to (38). In particular it is observed that the correlation under the $t$ distribution assumption approaches the correlation under the normal assumption for increased values of $v$. Note the restriction of $m_{1} \leq m_{2}$. Furthermore, it is seen that for varying $m_{1}$ that the correlation increases, as well as $\rho_{U_{1}, U_{2}}=0$ when $m_{1}=0$. This is seemingly evident because the bivariate nature of the bivariate gamma type distribution collapses when $m_{1}=0$, due to the construction discussed at the start of Section 3 . 
The cdf of the bivariate gamma type distribution with pdf (18) is of particular interest within wireless systems, and is given by

$$
\begin{aligned}
F\left(u_{1}, u_{2}\right)= & (1-\rho)^{m_{2}} \sum_{k=0}^{\infty} \sum_{l=0}^{\infty} \frac{\left(m_{1}\right)_{k}\left(m_{2}-m_{1}\right)_{l}}{\left(m_{2}+k\right)_{l} k ! l !} \frac{\rho^{k+l}}{\Gamma\left(m_{1}+k\right) \Gamma\left(m_{2}+k\right)} \\
& \times \int_{0}^{\infty} \gamma\left(m_{1}+k, \frac{t}{\Omega_{1}(1-\rho)} u_{1}\right) \gamma\left(m_{2}+k+l, \frac{t}{\Omega_{2}(1-\rho)} u_{2}\right) \mathcal{W}(t) d t
\end{aligned}
$$

where $m_{1}, m_{2}, \Omega_{1}, \Omega_{2}>0,-1<\rho<1, m_{2} \geq m_{1}$ and where $\gamma(\cdot, \cdot)$ denotes the lower incomplete gamma function (see [8]). This will be further explored in Section 5 .

Within a wireless communications framework, bivariate distributions with a LOS assumption are often employed to model land mobile satellite experiments. In particular, [11] emphasize that more sophisticated probability distributions are recalled to fit the behaviour of fading signals in more intricate scenarios. From a statistical viewpoint and origin, [16] showed that the noncentral chi-square distribution with $n$ degrees of freedom and noncentrality parameter $\theta$ can be represented as a weighted sum of univariate chi-square probabilities with weights equal to the probabilities of a Poisson distribution with expected value $\frac{\theta}{2}$. The genesis of the extension to the noncentral counterpart of the bivariate gamma type distribution with pdf (18) proposed here originated from the papers by [24], [23], and [7].

The proposed methodology, amended from the methodology for obtaining bivariate noncentral chi-square distributions by [7], to obtain bivariate noncentral gamma distributions is given as follows:

A bivariate noncentral gamma pdf can be obtained from a conditional bivariate central gamma distribution with pdf $f\left(u_{1}, u_{2} \mid k_{1}, k_{2}\right)$ in the following manner:

$$
f^{n c}\left(u_{1}, u_{2}\right)=\sum_{k_{1}=0}^{\infty} \sum_{k_{2}=0}^{\infty} f\left(u_{1}, u_{2} \mid k_{1}, k_{2}\right) g\left(k_{1}\right) g\left(k_{2}\right)
$$

where $g\left(k_{i}\right)=\frac{e^{-\frac{\theta_{i}}{2}}\left(\frac{\theta_{i}}{2}\right)^{k_{i}}}{k_{i} !}, i=1,2$ are Poisson probabilities where $\frac{\theta_{i}}{2}>0$ denotes the noncentrality parameters, and $f\left(u_{1}, u_{2} \mid k_{1}, k_{2}\right)$ the pdf of a bivariate gamma distribution with conditions on the shape parameters respectively. In this regard, the Laplace transform of $\left(U_{1}, U_{2}\right)$ in the noncentral case can be written as:

$$
\mathcal{L}_{U_{1}, U_{2}}^{n c}\left(s_{1}, s_{2}\right)=\sum_{k_{1}=0}^{\infty} \sum_{k_{2}=0}^{\infty} \mathcal{L}_{U_{1}, U_{2}}\left(s_{1}, s_{2} \mid k_{1}, k_{2}\right) g\left(k_{1}\right) g\left(k_{2}\right)
$$

where $\mathcal{L}_{U_{1}, U_{2}}\left(s_{1}, s_{2} \mid k_{1}, k_{2}\right)$ denotes the conditional Laplace transform of $\left(U_{1}, U_{2} \mid k_{1}, k_{2}\right)$ with pdf $f\left(u_{1}, u_{2} \mid k_{1}, k_{2}\right)$.

By conditioning on the shape parameters, $m_{1}$ and $m_{2}$, of the pdf in (18), we have:

$$
\begin{aligned}
f\left(u_{1}, u_{2} \mid t, k_{1}, k_{2}\right)= & (1-\rho)^{m_{2}+k_{2}} \sum_{k=0}^{\infty} \frac{\left(m_{1}+k_{1}\right)_{k}}{k !} \rho^{k}\left(\frac{1}{\Omega_{1}(1-\rho)}\right)^{m_{1}+k+k_{1}}\left(\frac{1}{\Omega_{2}(1-\rho)}\right)^{m_{2}+k+k_{2}} \\
& \times \frac{u_{1}^{m_{1}+k+k_{1}-1}}{\Gamma\left(m_{1}+k+k_{1}\right)} \frac{u_{2}^{m_{2}+k+k_{2}-1}}{\Gamma\left(m_{2}+k+k_{2}\right)} \exp \left[-t\left(\frac{u_{1}}{\Omega_{1}(1-\rho)}+\frac{u_{2}}{\Omega_{2}(1-\rho)}\right)\right] \\
& \times{ }_{1} F_{1}\left(m_{2}+k_{2}-m_{1}-k_{1}, m_{2}+k+k_{2} ; \frac{t \rho}{\Omega_{2}(1-\rho)} u_{2}\right) t^{m_{1}+m_{2}+2 k+k_{1}+k_{2}}
\end{aligned}
$$


for $u_{1}, u_{2}>0$ and where $m_{1}, m_{2}, \Omega_{1}, \Omega_{2}>0,-1<\rho<1$, and $m_{2} \geq m_{1}$. This means that the pdf of a bivariate noncentral gamma distribution can be constructed by substituting (25) into (23).

By deriving the Laplace transform, the noncentral counterpart of this bivariate gamma type distribution (18) becomes evident. From (24) and (25), and using [8] p. 346, eq. 3.381.4 and p. 815, eq. 7.522.9 follows:

$$
\begin{aligned}
& \mathcal{L}_{U_{1}, U_{2}}\left(s_{1}, s_{2} \mid t, k_{1}, k_{2}\right) \\
= & (1-\rho)^{m_{2}+k_{2}}\left(\frac{\frac{t}{\Omega_{1}(1-\rho)}}{\frac{t}{\Omega_{1}(1-\rho)}+s_{1}}\right)^{m_{1}+k_{1}}\left(\frac{\frac{t}{\Omega_{2}(1-\rho)}}{\frac{t}{\Omega_{2}(1-\rho)}+s_{2}}\right)^{m_{2}+k_{2}} \sum_{k=0}^{\infty} \frac{\left(m_{1}+k_{1}\right)_{k}}{k !} \\
& \times\left(\frac{\frac{t}{\Omega_{1}(1-\rho)}}{\frac{t}{\Omega_{1}(1-\rho)}+s_{1}}\right)^{k}\left(\frac{\frac{t \rho}{\Omega_{2}(1-\rho)}}{\frac{t}{\Omega_{2}(1-\rho)}+s_{2}}\right)^{k} \sum_{l=0}^{\infty} \frac{\left(m_{2}+k_{2}-m_{1}-k_{1}\right)_{l}}{l !}\left(\frac{\frac{t \rho}{\Omega_{2}(1-\rho)}}{\frac{t}{\Omega_{2}(1-\rho)}+s_{2}}\right)^{l} .
\end{aligned}
$$

Substituting (26) into (24) and after simplification:

$$
\begin{aligned}
& \mathcal{L}_{U_{1}, U_{2}}^{n c}\left(s_{1}, s_{2}\right) \\
& =\int_{0}^{\infty} \mathcal{L}_{U_{1}, U_{2}}\left(s_{1}, s_{2} \mid t\right) \mathcal{W}(t) d t \\
& =\int_{0}^{\infty}(1-\rho)^{-m_{1}}\left(\Omega_{1} t^{-1}\right)^{-m_{1}}\left(\Omega_{2} t^{-1}\right)^{-m_{2}} \\
& \times\left(\left(\frac{1}{\Omega_{1} t^{-1}(1-\rho)}+s_{1}\right)\left(\frac{1}{\Omega_{2} t^{-1}(1-\rho)}+s_{2}\right)-\left(\frac{\rho}{\Omega_{1} t^{-1} \Omega_{2} t^{-1}(1-\rho)^{2}}\right)\right)^{-m_{1}}\left(\frac{1}{\Omega_{2} t^{-1}}+s_{2}\right)^{-\left(m_{2}-m_{1}\right)} \\
& \times \exp \left(-\frac{\theta_{1}}{2}\right) \exp \left(\frac{\left(\frac{1}{\Omega_{2} t^{-1}}+s_{2}\right) \theta_{1}}{2(1-\rho)\left(\Omega_{1} t^{-1}\right)\left(\left(\frac{1}{\Omega_{1} t^{-1}(1-\rho)}+s_{1}\right)\left(\frac{1}{\Omega_{2} t^{-1}(1-\rho)}+s_{2}\right)-\frac{\rho}{\Omega_{1} t^{-1} \Omega_{2} t^{-1}(1-\rho)^{2}}\right)}\right) \\
& \times \exp \left(-\frac{\theta_{2}}{2}\right) \exp \left(\frac{\theta_{2}}{2\left(\left(\Omega_{2} t^{-1}\right)\left(\frac{1}{\Omega_{2} t^{-1}}+s_{2}\right)\right)}\right) \mathcal{W}(t) d t \text {. }
\end{aligned}
$$

Note that if $\theta_{1}=\theta_{2}=0$, (27) simplifies to the Laplace transform of the bivariate gamma type distribution (see (16)). Therefore, the pdf of a bivariate noncentral gamma type distribution is obtained by substituting (25) into (23), with expressions (42) and (43) for weights (2) and (3) respectively (see Appendix).

\section{Bivariate Weibullised gamma type distribution and characteristics}

In this section, a bivariate Weibullised gamma type distribution which emanates from (18) is of interest together with some properties. Suppose that $\left(U_{1}, U_{2}\right)$ is bivariate gamma type distributed with pdf (18), then the pdf of 
$\left(W_{1}, W_{2}\right)$, where $W_{i}=\left(\frac{U_{i}}{m_{i}}\right)^{\frac{1}{\beta_{i}}}$ is given by

$$
\begin{aligned}
f\left(w_{1}, w_{2}\right)= & \beta_{1} \beta_{2}(1-\rho)^{m_{2}} \sum_{k=0}^{\infty} \frac{\left(m_{1}\right)_{k} \rho^{k}}{k !}\left(\frac{m_{1}}{\Omega_{1}(1-\rho)}\right)^{m_{1}+k}\left(\frac{m_{2}}{\Omega_{2}(1-\rho)}\right)^{m_{2}+k} \\
& \times \frac{w_{1}^{\beta_{1} m_{1}+\beta_{1} k-1}}{\Gamma\left(m_{1}+k\right)} \frac{w_{2}^{\beta_{2} m_{2}+\beta_{2} k-1}}{\Gamma\left(m_{2}+k\right)} \int_{0}^{\infty} t^{m_{1}+m_{2}+2 k} \exp \left[-t\left(\frac{m_{1} w_{1}^{\beta_{1}}}{\Omega_{1}(1-\rho)}\right)\right] \\
& \times \exp \left[-t\left(\frac{m_{2} w_{2}^{\beta_{2}}}{\Omega_{2}(1-\rho)}\right)\right]{ }_{1} F_{1}\left(m_{2}-m_{1}, m_{2}+k ; \frac{m_{2} \rho t}{\Omega_{2}(1-\rho)} w_{2}^{\beta_{2}}\right) \mathcal{W}(t) d t
\end{aligned}
$$

for $w_{1}, w_{2}>0$ and where $m_{1}, m_{2}, \Omega_{1}, \Omega_{2}, \beta_{1}, \beta_{2}>0,-1<\rho<1$, and $m_{2} \geq m_{1}$, we refer to it as a bivariate Weibullised gamma type distribution. When $\beta_{1}=\beta_{2}=2$, then the distribution simplifies to a bivariate Nakagami type distribution, of which the bivariate Nakagami distribution in [18] is a special case of.

For weights (2) and (3) respectively, the results are given in Appendix equations (44) and (45). The contourplots of these specific cases are illustrated for $m_{1}=10, m_{2}=12, \Omega_{1}=3.5, \Omega_{2}=1.5, \beta_{1}=2, \rho=0.5$, and arbitrary choices for other parameters.
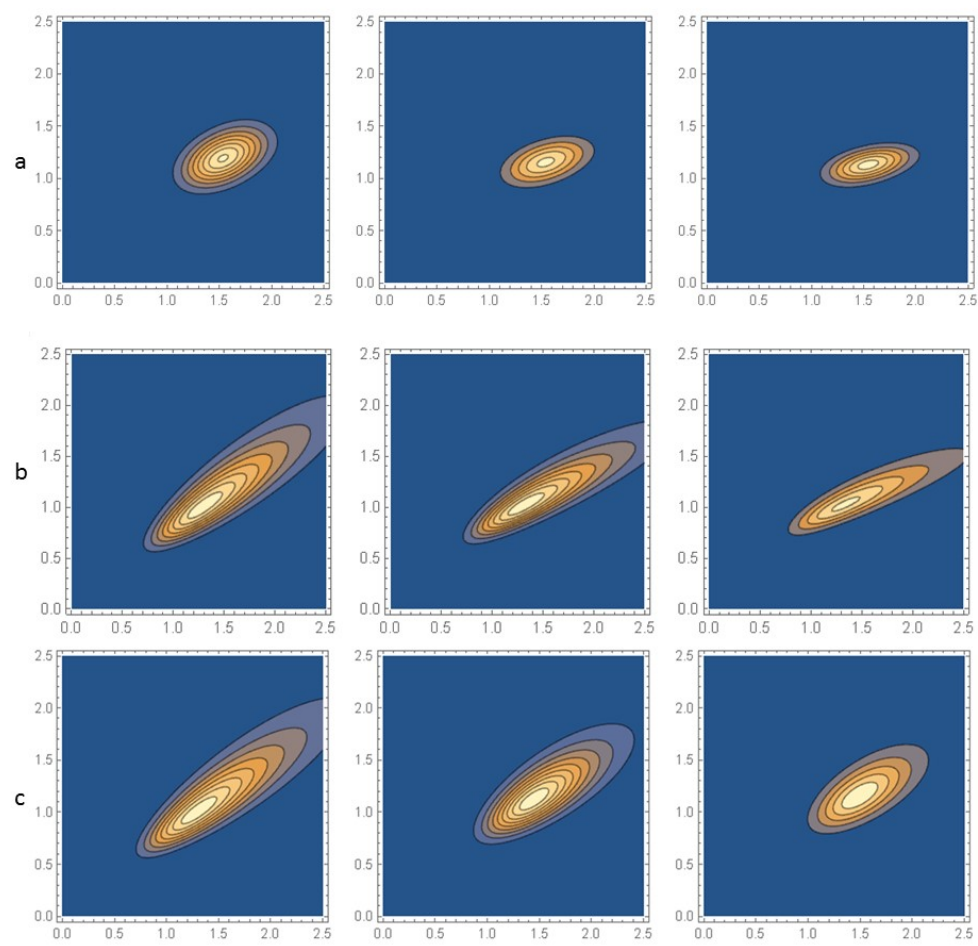

\section{Figure 3}

(a) Pdf (44) for $\beta_{2}=2,4,6$ (fltr).

(b) Pdf (45) for $v=5$ and $\beta_{2}=2,4,6$ (fltr).

(c) Pdf (45) for $\beta_{1}=\beta_{2}=2$ and varying $v=5,15,30$ (fltr). 
Figure 3 ((a) and (b)) illustrate the effect of different values of $\beta_{2}$; particularly also noting the fatter tails in the contour of (45) as compared to (44). Figure 3 (c) indicate the effect on the tails for increasing values of $v$ for the bivariate Nakagami type distribution with weight (3).

The Laplace transform of the variables under which a fading channel is operating can be used to evaluate certain attributes of a wireless system, such as the average bit error rate (see [20], [21]). Thus the Laplace transform of $\left(W_{1}, W_{2}\right)$ with pdf (28) is given as:

$$
\begin{aligned}
& \mathcal{L}_{W_{1}, W_{2}}\left(s_{1}, s_{2}\right) \\
& =(2 \pi)^{\frac{1}{2}\left(1-\beta_{1}\right)}(1-\rho)^{m_{2}} \sum_{k=0}^{\infty} \sum_{l=0}^{\infty} \frac{\left(m_{1}\right)_{k} \rho^{k}}{k ! l !}\left(\frac{m_{1}}{\Omega_{1}(1-\rho)}\right)^{m_{1}+k}\left(\frac{m_{2}}{\Omega_{2}(1-\rho)}\right)^{-\left(\frac{l}{\beta_{2}}\right)} \frac{\Gamma\left(m_{2}+k+\frac{l}{\beta_{2}}\right)}{\Gamma\left(m_{1}+k\right) \Gamma\left(m_{2}+k\right)} \\
& \times \beta_{1}^{\beta_{1} m_{1}+\beta_{1} k-\frac{1}{2}} s_{1}^{-\beta_{1} m_{1}-\beta_{1} k}\left(-s_{2}\right)^{l}{ }_{2} F_{1}\left(m_{2}-m_{1}, m_{2}+k+\frac{l}{\beta_{2}}, m_{2}+k ; \rho\right)
\end{aligned}
$$

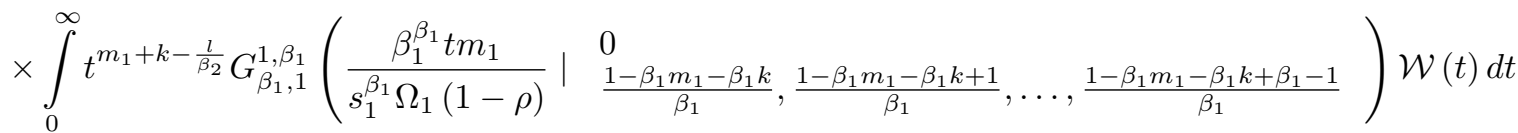

where $m_{1}, m_{2}, \Omega_{1}, \Omega_{2}, \beta_{1}, \beta_{2}>0,-1<\rho<1, m_{2} \geq m_{1}$, and $G_{p, q}^{m, n}\left(x \mid a_{1}, \ldots, a_{p} b_{1}, \ldots, b_{p}\right)$ denotes Meijer's $G$ function (see [12]).

Specific cases for the weights (2) and (3) of (29) are given in the Appendix as equations (46) and (47) respectively, and particular values are evaluated; the machine runtime is listed as well. Furthermore, the integral representation (definition) of the Laplace transform was also calculated numerically for the normal- and $t$ case ((29) for weights in (2) and (3)), together with its machine runtime. Similar as in [19], the calculated values of (46), (47), and integral definition for both normal- and $t$ cases for select values of $s_{1}$ and $s_{2}$ are provided. The runtime is computed using Mathematica 11 on an Intel i7 processor. Whilst the runtime is affected by various factors of an individual processor, it gives some indication about the speed with which these expressions may be evaluated. The parameters for which these values are calculated are $m_{1}=10, m_{2}=12, \Omega_{1}=2.5, \Omega_{2}=1.5, \rho=0.5, v=5$, and $\beta_{1}=\beta_{2}=2$.

\begin{tabular}{|l|l|l|l|l|l|}
\hline & & Normal & t & \multicolumn{1}{l|}{$\begin{array}{l}\text { Runtime } \\
\text { (seconds) }\end{array}$} \\
\cline { 3 - 6 } & & Value & $\begin{array}{c}\text { Runtime } \\
\text { (seconds) }\end{array}$ & Value & 12.6415 \\
\hline $\mathcal{L}_{W_{1}, W_{2}}(0.5,0.5)$ & $(46),(47)$ & 0.125162 & 153.0130 & 0.113083 & 12.0136 \\
\cline { 2 - 6 } & Integral & 0.126973 & 77.5169 & 0.121326 & 576.86 \\
\hline $\mathcal{L}_{W_{1}, W_{2}}(0.5,1)$ & $(46),(47)$ & 0.0695437 & 153.153 & 0.0665175 & 12.2351 \\
\cline { 2 - 6 } & Integral & 0.0704006 & 77.8445 & 0.0612938 & 475.085 \\
\hline $\mathcal{L}_{W_{1}, W_{2}}(1,1)$ & $(46),(47)$ & 0.0329823 & 156.377 & 0.0320912 & 12.7365 \\
\cline { 2 - 6 } & Integral & 0.0332989 & 75.3485 & 0.0269502 & 380.268 \\
\hline
\end{tabular}

Table 1 Comparison between Laplace integral- and derived expressions for normal and $t$ models

The following observations can be made from Table 1:

- The calculated values using expressions (46) and (47) illustrate the accuracy of computation when compared to integral definition; 
- Upon comparing the calculated values of (46) and (47), it is observed that the values of (47) are consistently lower than the counterparts under ((46));

- Using (47) versus numerically integrating the definition indicates significant shortening of required runtime; and

- The runtime of calculating (47) versus calculating (46) is significantly faster. is

For completeness' sake, The product moment of the bivariate Weibullised gamma type distribution with pdf (28)

$$
\begin{aligned}
E\left(W_{1}^{n} W_{2}^{j}\right)= & (1-\rho)^{m_{2}} \sum_{k=0}^{\infty} \sum_{l=0}^{\infty} \frac{\left(m_{1}\right)_{k}\left(m_{2}-m_{1}\right)_{l} \rho^{k+l}}{l ! k !\left(m_{2}+k\right)_{l}} \frac{1}{\Gamma\left(m_{1}+k\right) \Gamma\left(m_{2}+k\right)} \Gamma\left(\frac{n}{\beta_{1}}+m_{1}+k\right) \Gamma\left(\frac{j+l}{\beta_{2}}+m_{2}+k\right) \\
& \times\left(\frac{m_{1}}{\Omega_{1}(1-\rho)}\right)^{-\left(\frac{n}{\beta_{1}}\right)}\left(\frac{m_{2}}{\Omega_{2}(1-\rho)}\right)^{-\left(\frac{j+l}{\beta_{2}}-l\right)} \int_{0}^{\infty} t^{-\left(\frac{j+l}{\beta_{2}}+\frac{n}{\beta_{1}}-l\right)} \mathcal{W}(t) d t
\end{aligned}
$$

where $n, j>0$, and the cdf of the bivariate Weibullised gamma type distribution with pdf (28) is given by

$$
\begin{aligned}
F\left(w_{1}, w_{2}\right)= & (1-\rho)^{m_{2}} \sum_{k=0}^{\infty} \sum_{l=0}^{\infty} \frac{\left(m_{1}\right)_{k}\left(m_{2}-m_{1}\right)_{l}}{\left(m_{2}+k\right)_{l} k ! l !} \frac{\rho^{k+l}}{\Gamma\left(m_{1}+k\right) \Gamma\left(m_{2}+k\right)} \\
& \times \int_{0}^{\infty} \gamma\left(m_{1}+k, \frac{t m_{1}}{\Omega_{1}(1-\rho)} w_{1}^{\beta_{1}}\right) \gamma\left(m_{2}+k+l, \frac{t m_{2}}{\Omega_{2}(1-\rho)} w_{1}^{\beta_{2}}\right) \mathcal{W}(t) d t
\end{aligned}
$$

where $m_{2}, m_{1}, \Omega_{1}, \Omega_{2}, \beta_{1}, \beta_{2}>0,-1<\rho<1$, and $m_{2} \geq m_{1}$. Simplified results of (30) and (31) for weights (2) and (3) are given in the Appendix as equations (48) through (51).

\section{Illustrative application}

The maximum of a bivariate variable following a bivariate gamma type distribution such as (18) can be used to model the performance of a MIMO system subject to Rayleigh type fading (6) (see [17], [25]). The outage probability at a certain threshold is obtained as (see [21]):

$$
F(u)=P\left\{\max \left(U_{1}, U_{2}\right)<u\right\} .
$$

Using (22), the cdf of $\max \left(U_{1}, U_{2}\right)$ is:

$$
\begin{aligned}
F(u)= & (1-\rho)^{m_{2}} \sum_{k=0}^{\infty} \sum_{l=0}^{\infty} \frac{\left(m_{1}\right)_{k}\left(m_{2}-m_{1}\right)_{l}}{\left(m_{2}+k\right)_{l} k ! l !} \frac{\rho^{k+l}}{\Gamma\left(m_{1}+k\right) \Gamma\left(m_{2}+k\right)} \\
& \times \int_{0}^{\infty} \gamma\left(m_{1}+k, \frac{t}{\Omega_{1}(1-\rho)} u\right) \gamma\left(m_{2}+k+l, \frac{t}{\Omega_{2}(1-\rho)} u\right) \mathcal{W}(t) d t .
\end{aligned}
$$

By choosing $\mathcal{W}(t)$ as (2), the cdf is given by:

$$
\begin{aligned}
F(u)= & (1-\rho)^{m_{2}} \sum_{k=0}^{\infty} \sum_{l=0}^{\infty} \frac{\left(m_{1}\right)_{k}\left(m_{2}-m_{1}\right)_{l}}{\left(m_{2}+k\right)_{l} k ! l !} \frac{\rho^{k+l}}{\Gamma\left(m_{1}+k\right) \Gamma\left(m_{2}+k\right)} \\
& \times \gamma\left(m_{1}+k, \frac{1}{\Omega_{1}(1-\rho)} u\right) \gamma\left(m_{2}+k+l, \frac{1}{\Omega_{2}(1-\rho)} u\right) .
\end{aligned}
$$


By choosing $\mathcal{W}(t)$ as (3), the cdf is given by:

$$
\begin{aligned}
F(u)= & \frac{\left(\frac{v}{2}\right)^{\frac{v}{2}}}{\Gamma\left(\frac{v}{2}\right)}(1-\rho)^{m_{2}} \sum_{k=0}^{\infty} \sum_{l=0}^{\infty} \frac{\left(m_{1}\right)_{k}\left(m_{2}-m_{1}\right)_{l}}{\left(m_{2}+k\right)_{l} k ! l !} \frac{\rho^{k+l}}{\Gamma\left(m_{1}+k\right) \Gamma\left(m_{2}+k\right)} \\
& \times \int_{0}^{\infty} \gamma\left(m_{1}+k, \frac{t}{\Omega_{1}(1-\rho)} u\right) \gamma\left(m_{2}+k+l, \frac{t}{\Omega_{2}(1-\rho)} u\right) t^{\frac{v}{2}-1} \exp \left(-\frac{v t}{2}\right) d t
\end{aligned}
$$

respectively. These analytic expressions for the cdf of the maximum act as the expressions for the outage probability of the signal-to-noise ratio (SNR) for a MIMO system based on fading characteristics illustrated by the distribution with pdf (18). It is illustrated for arbitrary parameters, specifically, $m_{1}=10, m_{2}=12, \rho=0.5, \Omega_{1}=2.5, \Omega_{2}=$ 1.5 , and $v=5$.

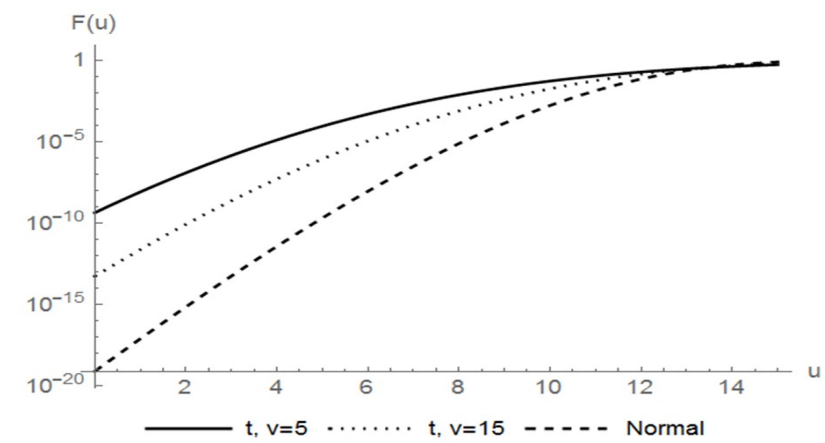

Figure 4 Analytical outage probabilities (32) and (33).

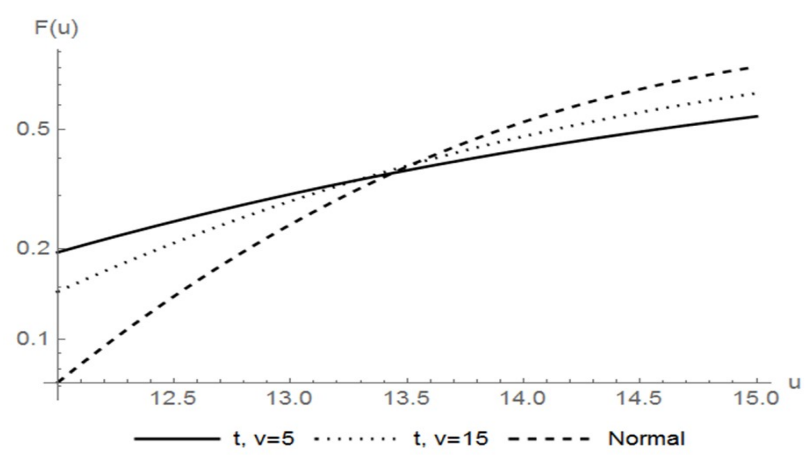

Figure 5 Analytical outage probabilities (32) and (33), zoomed in. 
Figures 4 and 5 illustrates that (32) exhibits a lower outage probability for small outage thresholds than (33). However, for the scenario where the outage threshold is large, (33) exhibits a lower outage probability. This observation provides significant insight to the theoretical contribution of the candidacy of the underlying $t$ distribution in comparison to the usual underlying normal case.

\section{Conclusions}

This paper proposes new bivariate distributions emanating from the elliptical distribution. In particular, a new bivariate gamma type distribution has been derived, along with its noncentral counterpart. Furthermore, a bivariate Weibullised gamma type distribution, stemming from the bivariate gamma type distribution, has also been derived and studied. Special cases, in particular for underlying normal- and underlying $t$ distribution, has been presented in the Appendix for derived results. Possible application has been demonstrated in terms of applying the newly derived results within the wireless communications field. In particular, the outage probability evaluation for wireless systems provides significant insight into the behaviour of the bivariate gamma type distribution, specifically with an underlying $t$ distribution, in such scenarios. Subsequent work would include investigating different members of the elliptical distributions as viable underlying distributions in wireless systems settings.

\section{Acknowledgements}

The authors acknowledge the support of the StatDisT group. This work is based on the research supported in part by the National Research Foundation of South Africa (SARChI Research Chair- UID: 71199; and Grant ref. CPRR160403161466 nr. 105840). Opinions expressed and conclusions arrived at are those of the author and are not necessarily to be attributed to the NRF.

\section{Appendix}

Detailed derivations of the results presented in the Appendix are available from the authors. Special cases of the derived results pertaining to weights (2) and (5) are presented in this section.

\subsection{Bivariate gamma type}

\subsection{1. $P d f$}

- By choosing $\mathcal{W}(t)$ as (2), (18) simplifies to the result of [18] (eq. 10):

$$
\begin{aligned}
f_{\text {normal }}\left(u_{1}, u_{2}\right)= & (1-\rho)^{m_{2}} \sum_{k=0}^{\infty} \frac{\left(m_{1}\right)_{k}}{k !} \rho^{k}\left(\frac{1}{\Omega_{1}(1-\rho)}\right)^{m_{1}+k}\left(\frac{1}{\Omega_{2}(1-\rho)}\right)^{m_{2}+k} \\
& \times \frac{u_{1}^{m_{1}+k-1}}{\Gamma\left(m_{1}+k\right)} \frac{u_{2}^{m_{2}+k-1}}{\Gamma\left(m_{2}+k\right)} \exp \left[-\frac{u_{1}}{\Omega_{1}(1-\rho)}\right] \exp \left[-\frac{u_{2}}{\Omega_{2}(1-\rho)}\right] \\
& \times{ }_{1} F_{1}\left(m_{2}-m_{1}, m_{2}+k ; \frac{\rho}{\Omega_{2}(1-\rho)} u_{2}\right)
\end{aligned}
$$

for $u_{1}, u_{2}>0$ and where $m_{1}, m_{2}, \Omega_{1}, \Omega_{2}>0,-1<\rho<1$, and $m_{2} \geq m_{1}$. 
- If $\mathcal{W}(t)$ is chosen as (3) and using [8], p. 815, eq. 7.522.9, (18) simplifies to:

$$
\begin{aligned}
f_{t}\left(u_{1}, u_{2}\right)= & \frac{\left(\frac{v}{2}\right)^{\frac{v}{2}}}{\Gamma\left(\frac{v}{2}\right)}(1-\rho)^{m_{2}} \sum_{k=0}^{\infty} \frac{\left(m_{1}\right)_{k}}{k !} \rho^{k}\left(\frac{1}{\Omega_{1}(1-\rho)}\right)^{m_{1}+k}\left(\frac{1}{\Omega_{2}(1-\rho)}\right)^{m_{2}+k} \frac{u_{1}^{m_{1}+k-1}}{\Gamma\left(m_{1}+k\right)} \\
& \times \frac{u_{2}^{m_{2}+k-1}}{\Gamma\left(m_{2}+k\right)} \Gamma\left(m_{1}+m_{2}+2 k+\frac{v}{2}\right)\left(\frac{u_{1}}{\Omega_{1}(1-\rho)}+\frac{u_{2}}{\Omega_{2}(1-\rho)}+\frac{v}{2}\right)^{-\left(m_{1}+m_{2}+2 k+\frac{v}{2}\right)} \\
& \times{ }_{2} F_{1}\left(m_{2}-m_{1}, m_{1}+m_{2}+2 k+\frac{v}{2} ; m_{2}+k ; \frac{\rho}{\Omega_{2}(1-\rho)\left(\frac{u_{1}}{\Omega_{1}(1-\rho)}+\frac{u_{2}}{\Omega_{2}(1-\rho)}+\frac{v}{2}\right)} u_{2}\right)(35)
\end{aligned}
$$

for $u_{1}, u_{2}>0$ and where $m_{1}, m_{2}, \Omega_{1}, \Omega_{2}>0,-1<\rho<1$, and $m_{2} \geq m_{1} \cdot{ }_{2} F_{1}(\cdot)$ denotes the Gauss hypergeometric function (see [8]), with restriction $\left|\frac{\rho}{\Omega_{2}(1-\rho)\left(\frac{u_{1}}{\Omega_{1}(1-\rho)}+\frac{u_{2}}{\Omega_{2}(1-\rho)}+\frac{v}{2}\right)} u_{2}\right|<1$.

\subsubsection{Marginals}

- By choosing $\mathcal{W}(t)$ as (2), (19) simplifies to:

$$
f\left(u_{i}\right)=\frac{u_{i}^{m_{i}-1}}{\Omega_{i}^{m_{i}} \Gamma\left(m_{i}\right)} \exp \left[-\left(\frac{u_{i}}{\Omega_{i}}\right)\right]
$$

for $u_{i}>0$ and $m_{i}, \Omega_{i}>0$. This distribution in (36) is easily identifiable as the well know two parameter gamma distribution.

- If $\mathcal{W}(t)$ is chosen as (3) and by using [8], p. 346, eq. 3.381.4, (19) simplifies to

$$
f\left(u_{i}\right)=\frac{\left(\frac{v}{2}\right)^{\frac{v}{2}}}{B\left(\frac{v}{2}, m_{i}\right) \Omega_{i}^{m_{i}}} \frac{u_{i}^{m_{i}-1}}{\left(\frac{u_{i}}{\Omega_{i}}+\frac{v}{2}\right)^{m_{i}+\frac{v}{2}}}
$$

for $u_{i}>0$ and $m_{i}, \Omega_{i}, v>0$, and where $B(\cdot, \cdot)$ denotes the beta function.

\subsubsection{Product moments}

- By choosing $\mathcal{W}(t)$ as (2), (20) simplifies to:

$$
\begin{aligned}
E\left(U_{1}^{n} U_{2}^{j}\right)= & \Omega_{1}^{n} \Omega_{2}^{j}(1-\rho)^{n+j+m_{2}} \sum_{k=0}^{\infty} \frac{\left(m_{1}\right)_{k}}{k !} \rho^{k} \\
& \times \frac{\Gamma\left(n+m_{1}+k\right) \Gamma\left(j+m_{2}+k\right)}{\Gamma\left(m_{1}+k\right) \Gamma\left(m_{2}+k\right)}{ }_{2} F_{1}\left(m_{2}-m_{1}, j+m_{2}+k ; m_{2}+k ; \rho\right)
\end{aligned}
$$

where $n, j>0$, and $m_{2}, m_{1}, \Omega_{1}, \Omega_{2}>0,-1<\rho<1$, and $m_{2} \geq m_{1}$.

- By choosing $\mathcal{W}(t)$ as (3) and using [8], p. 346, eq. 3.381.4, (20) simplifies to:

$$
\begin{aligned}
E\left(U_{1}^{n} U_{2}^{j}\right)= & \frac{\left(\frac{v}{2}\right)^{n+j} \Gamma\left(\frac{v}{2}-(n+j)\right)}{\Gamma\left(\frac{v}{2}\right)} \Omega_{1}^{n} \Omega_{2}^{j}(1-\rho)^{n+j+m_{2}} \sum_{k=0}^{\infty} \frac{\left(m_{1}\right)_{k}}{k !} \rho^{k} \\
& \times \frac{\Gamma\left(n+m_{1}+k\right) \Gamma\left(j+m_{2}+k\right)}{\Gamma\left(m_{1}+k\right) \Gamma\left(m_{2}+k\right)}{ }_{2} F_{1}\left(m_{2}-m_{1}, j+m_{2}+k ; m_{2}+k ; \rho\right)
\end{aligned}
$$

and where $n, j>0, m_{2}, m_{1}, \Omega_{1}, \Omega_{2}, v>0,-1<\rho<1$, and $m_{2} \geq m_{1}$. Note a particular restriction, $\Gamma\left(\frac{v}{2}-(n+j)\right)$ implies that $v>2(n+j)$ in order for the gamma function to be well defined. 


\subsection{4. $C d f$}

- By choosing $\mathcal{W}(t)$ as (2), (22) simplifies to:

$$
\begin{aligned}
F_{\text {normal }}\left(u_{1}, u_{2}\right)= & (1-\rho)^{m_{2}} \sum_{k=0}^{\infty} \sum_{l=0}^{\infty} \frac{\left(m_{1}\right)_{k}\left(m_{2}-m_{1}\right)_{l}}{\left(m_{2}+k\right)_{l} k ! l !} \frac{\rho^{k+l}}{\Gamma\left(m_{1}+k\right) \Gamma\left(m_{2}+k\right)} \\
& \times \gamma\left(m_{1}+k, \frac{1}{\Omega_{1}(1-\rho)} u_{1}\right) \gamma\left(m_{2}+k+l, \frac{1}{\Omega_{2}(1-\rho)} u_{2}\right)
\end{aligned}
$$

where $m_{1}, m_{2}, \Omega_{1}, \Omega_{2}>0,-1<\rho<1$, and $m_{2} \geq m_{1}$.

- By choosing $\mathcal{W}(t)$ as (3), (22) simplifies to:

$$
\begin{aligned}
F_{t}\left(u_{1}, u_{2}\right)= & \frac{\left(\frac{v}{2}\right)^{\frac{v}{2}}}{\Gamma\left(\frac{v}{2}\right)}(1-\rho)^{m_{2}} \sum_{k=0}^{\infty} \sum_{l=0}^{\infty} \frac{\left(m_{1}\right)_{k}\left(m_{2}-m_{1}\right)_{l}}{\left(m_{2}+k\right)_{l} k ! l !} \frac{\rho^{k+l}}{\Gamma\left(m_{1}+k\right) \Gamma\left(m_{2}+k\right)} \\
& \times \int_{0}^{\infty} \gamma\left(m_{1}+k, \frac{t}{\Omega_{1}(1-\rho)} u_{1}\right) \gamma\left(m_{2}+k+l, \frac{t}{\Omega_{2}(1-\rho)} u_{2}\right) t^{\frac{v}{2}-1} \exp \left(-\frac{v t}{2}\right) d t
\end{aligned}
$$

where $m_{1}, m_{2}, \Omega_{1}, \Omega_{2}, v>0,-1<\rho<1$, and $m_{2} \geq m_{1}$.

\subsubsection{Noncentral counterpart}

- By choosing $\mathcal{W}(t)$ as (2), (23) simplifies to:

$$
\begin{aligned}
= & \sum_{k_{1}=0}^{\infty} \sum_{k_{2}=0}^{\infty}(1-\rho)^{m_{2}+k_{2}} \sum_{k=0}^{\infty} \frac{\left(m_{1}+k_{1}\right)_{k}}{k !} \rho^{k}\left(\frac{1}{\Omega_{1}(1-\rho)}\right)^{m_{1}+k+k_{1}}\left(\frac{1}{\Omega_{2}(1-\rho)}\right)^{m_{2}+k+k_{2}} \\
& \times \frac{u_{1}^{m_{1}+k+k_{1}-1}}{\Gamma\left(m_{1}+k+k_{1}\right)} \frac{u_{2}^{m_{2}+k+k_{2}-1}}{\Gamma\left(m_{2}+k+k_{2}\right)} \exp \left[-\left(\frac{u_{1}}{\Omega_{1}(1-\rho)}+\frac{u_{2}}{\Omega_{2}(1-\rho)}\right)\right] \\
& \times{ }_{1} F_{1}\left(m_{2}+k_{2}-m_{1}-k_{1}, m_{2}+k+k_{2} ; \frac{\rho}{\Omega_{2}(1-\rho)} u_{2}\right) \frac{\exp \left(-\frac{\theta_{1}}{2}\right)\left(\frac{\theta_{1}}{2}\right)^{k_{1}}}{k_{1} !} \frac{\exp \left(-\frac{\theta_{2}}{2}\right)\left(\frac{\theta_{2}}{2}\right)^{k_{2}}}{k_{2} !}
\end{aligned}
$$

- By choosing $\mathcal{W}(t)$ as (3), (23) simplifies to:

$$
\begin{aligned}
& f_{t}^{n c}\left(u_{1}, u_{2}\right) \\
= & \frac{\left(\frac{v}{2}\right)^{\frac{v}{2}}}{\Gamma\left(\frac{v}{2}\right)} \sum_{k=0}^{\infty} \sum_{k_{1}=0}^{\infty} \sum_{k_{2}=0}^{\infty}(1-\rho)^{m_{2}+k_{2}} \frac{\left(m_{1}+k_{1}\right)_{k}}{k !} \rho^{k}\left(\frac{1}{\Omega_{1}(1-\rho)}\right)^{m_{1}+k+k_{1}}\left(\frac{1}{\Omega_{2}(1-\rho)}\right)^{m_{2}+k+k_{2}} \\
& \times \frac{u_{1}^{m_{1}+k+k_{1}-1}}{\Gamma\left(m_{1}+k+k_{1}\right)} \frac{u_{2}^{m_{2}+k+k_{2}-1}}{\Gamma\left(m_{2}+k+k_{2}\right)} \frac{\exp \left(-\frac{\theta_{1}}{2}\right)\left(\frac{\theta_{1}}{2}\right)^{k_{1}}}{k_{1} !} \frac{\exp \left(-\frac{\theta_{2}}{2}\right)\left(\frac{\theta_{2}}{2}\right)^{k_{2}}}{k_{2} !} \\
& \times \Gamma\left(m_{1}+m_{2}+2 k+k_{1}+k_{2}+\frac{v}{2}\right)\left(\frac{u_{1}}{\Omega_{1}(1-\rho)}+\frac{u_{2}}{\Omega_{2}(1-\rho)}+\frac{v}{2}\right)^{-\left(m_{1}+m_{2}+2 k+k_{1}+k_{2}+\frac{v}{2}\right)} \\
& \times{ }_{2} F_{1}\left(m_{2}+k_{2}-m_{1}-k_{1}, m_{1}+m_{2}+2 k+k_{1}+k_{2}+\frac{v}{2}\right. \\
& \left.m_{2}+k+k_{2} ; \frac{\rho}{\Omega_{2}(1-\rho)\left(\frac{u_{1}}{\Omega_{1}(1-\rho)}+\frac{u_{2}}{\Omega_{2}(1-\rho)}+\frac{v}{2}\right)} u_{2}\right)
\end{aligned}
$$




\subsection{Bivariate Weibullised gamma type}

\subsection{1. $P d f$}

- By choosing $\mathcal{W}(t)$ as (2), (28) simplifies to:

$$
\begin{aligned}
f_{\text {normal }}\left(w_{1}, w_{2}\right)= & \beta_{1} \beta_{2}(1-\rho)^{m_{2}} \sum_{k=0}^{\infty} \frac{\left(m_{1}\right)_{k} \rho^{k}}{k !}\left(\frac{m_{1}}{\Omega_{1}(1-\rho)}\right)^{m_{1}+k}\left(\frac{m_{2}}{\Omega_{2}(1-\rho)}\right)^{m_{2}+k} \\
& \times \frac{w_{1}^{\beta_{1} m_{1}+\beta_{1} k-1}}{\Gamma\left(m_{1}+k\right)} \frac{w_{2}^{\beta_{2} m_{2}+\beta_{2} k-1}}{\Gamma\left(m_{2}+k\right)} \exp \left[-\frac{m_{1} w_{1}^{\beta_{1}}}{\Omega_{1}(1-\rho)}\right] \exp \left[-\frac{m_{2} w_{2}^{\beta_{2}}}{\Omega_{2}(1-\rho)}\right] \\
& \times{ }_{1} F_{1}\left(m_{2}-m_{1}, m_{2}+k ; \frac{m_{2} \rho}{\Omega_{2}(1-\rho)} w_{2}^{\beta_{2}}\right)
\end{aligned}
$$

where $m_{1}, m_{2}, \Omega_{1}, \Omega_{2}>0,-1<\rho<1$, and $m_{2} \geq m_{1}$.

- By choosing $\mathcal{W}(t)$ as (3), (28) simplifies to:

$$
\begin{aligned}
f_{t}\left(w_{1}, w_{2}\right)= & \frac{\left(\frac{v}{2}\right)^{\frac{v}{2}}}{\Gamma\left(\frac{v}{2}\right)} \beta_{1} \beta_{2}(1-\rho)^{m_{2}} \sum_{k=0}^{\infty} \frac{\left(m_{1}\right)_{k} \rho^{k}}{k !}\left(\frac{m_{1}}{\Omega_{1}(1-\rho)}\right)^{m_{1}+k}\left(\frac{m_{2}}{\Omega_{2}(1-\rho)}\right)^{m_{2}+k} \\
& \times \frac{w_{1}^{\beta_{1} m_{1}+\beta_{1} k-1}}{\Gamma\left(m_{1}+k\right)} \frac{w_{2}^{\beta_{2} m_{2}+\beta_{2} k-1}}{\Gamma\left(m_{2}+k\right)} \Gamma\left(m_{1}+m_{2}+2 k+\frac{v}{2}\right)\left(\frac{m_{1} w_{1}^{\beta_{1}}}{\Omega_{1}(1-\rho)}+\frac{m_{2} w_{2}^{\beta_{2}}}{\Omega_{2}(1-\rho)}+\frac{v}{2}\right)^{-\left(m_{1}+m_{2}+2 k+\frac{v}{2}\right)} \\
& \times{ }_{2} F_{1}\left(m_{2}-m_{1}, m_{1}+m_{2}+2 k+\frac{v}{2} ; m_{2}+k ; \frac{\frac{m_{2} w_{2}^{\beta_{2}}}{\Omega_{2}(1-\rho)}}{\left(\frac{m_{1} w_{1}^{\beta_{1}}}{\Omega_{1}(1-\rho)}+\frac{m_{2} w_{2}^{\beta_{2}}}{\Omega_{2}(1-\rho)}+\frac{v}{2}\right)} \rho\right)
\end{aligned}
$$

where $m_{1}, m_{2}, \Omega_{1}, \Omega_{2}, v>0,-1<\rho<1$, and $m_{2} \geq m_{1}$. As before, note the restriction of the Gauss hypergeometric function such that $\left|\frac{\frac{m_{2} w_{2}^{\beta_{2}}}{\Omega_{2}(1-\rho)}}{\left(\frac{m_{1} w_{1}^{\beta_{1}}}{\Omega_{1}(1-\rho)}+\frac{m_{2} w_{2}^{\beta_{2}}}{\Omega_{2}(1-\rho)}+\frac{v}{2}\right)}\right|<1$.

\subsubsection{Laplace transform}

- By choosing $\mathcal{W}(t)$ as (2), (29) simplifies to:

$$
\begin{aligned}
& \mathcal{L}_{\text {normal }}\left(s_{1}, s_{2}\right) \\
= & (1-\rho)^{m_{2}} \sum_{k=0}^{\infty} \sum_{l=0}^{\infty} \frac{\left(m_{1}\right)_{k} \rho^{k}}{k ! l !}\left(\frac{m_{1}}{\Omega_{1}(1-\rho)}\right)^{m_{1}+k}\left(\frac{m_{2}}{\Omega_{2}(1-\rho)}\right)^{-\left(\frac{l}{\beta_{2}}\right)} \frac{\Gamma\left(m_{2}+k+\frac{l}{\beta_{2}}\right)}{\Gamma\left(m_{1}+k\right) \Gamma\left(m_{2}+k\right)}(2 \pi)^{\frac{1}{2}\left(1-\beta_{1}\right)} \\
& \times \beta_{1}^{\beta_{1} m_{1}+\beta_{1} k-\frac{1}{2}} s_{1}^{-\beta_{1} m_{1}-\beta_{1} k}\left(-s_{2}\right)_{2}^{l} F_{1}\left(m_{2}-m_{1}, m_{2}+k+\frac{l}{\beta_{2}}, m_{2}+k ; \rho\right) \\
& \times G_{\beta_{1}, 1}^{1, \beta_{1}}\left(\frac{\beta_{1}^{\beta_{1}} m_{1}}{s_{1}^{\beta_{1}} \Omega_{1}(1-\rho)} \mid \stackrel{0}{\frac{1-\beta_{1} m_{1}-\beta_{1} k}{\beta_{1}}}, \frac{1-\beta_{1} m_{1}-\beta_{1} k+1}{\beta_{1}}, \ldots, \frac{1-\beta_{1} m_{1}-\beta_{1} k+\beta_{1}-1}{\beta_{1}}\right)
\end{aligned}
$$

where $m_{1}, m_{2}, \Omega_{1}, \Omega_{2}>0-1<\rho<1$, and $m_{2} \geq m_{1}$. 
- By choosing $\mathcal{W}(t)$ as (3) and using [12], p. 80, eq. 3.2.2, (29) simplifies to:

$$
\begin{aligned}
& \mathcal{L}_{t}\left(s_{1}, s_{2}\right) \\
& =\frac{(1-\rho)^{m_{2}}}{\Gamma\left(\frac{v}{2}\right)} \sum_{k=0}^{\infty} \sum_{l=0}^{\infty} \frac{\left(m_{1}\right)_{k} \rho^{k}}{k ! l !}\left(\frac{m_{1}}{\Omega_{1}(1-\rho)}\right)^{m_{1}+k}\left(\frac{m_{2}}{\Omega_{2}(1-\rho)}\right)^{-\left(\frac{l}{\beta_{2}}\right)} \frac{\Gamma\left(m_{2}+k+\frac{l}{\beta_{2}}\right)}{\Gamma\left(m_{1}+k\right) \Gamma\left(m_{2}+k\right)} \\
& \times(2 \pi)^{\frac{1}{2}\left(1-\beta_{1}\right)} \beta_{1}^{\beta_{1} m_{1}+\beta_{1} k-\frac{1}{2}} s_{1}^{-\beta_{1} m_{1}-\beta_{1} k}\left(-s_{2}\right)^{l}{ }_{2} F_{1}\left(m_{2}-m_{1}, m_{2}+k+\frac{l}{\beta_{2}}, m_{2}+k ; \rho\right)\left(\frac{v}{2}\right)^{-\left(m_{1}+k-\frac{l}{\beta_{2}}\right)} \\
& \times G_{\beta_{1}+1,1}^{1, \beta_{1}+1}\left(\begin{array}{lll}
\frac{\beta_{1}^{\beta_{1}} m_{1}}{s_{1}^{\beta_{1}} \Omega_{1}(1-\rho) \frac{v}{2}} \mid & 0 \\
\frac{1-\beta_{1} m_{1}-\beta_{1} k}{\beta_{1}} & \frac{1-\beta_{1} m_{1}-\beta_{1} k+1}{\beta_{1}}, \ldots, \frac{1-\beta_{1} m_{1}-\beta_{1} k+\beta_{1}-1}{\beta_{1}}, 1-\left(m_{1}+k+\frac{v}{2}-\frac{l}{\beta_{2}}\right)
\end{array}\right) .
\end{aligned}
$$

\subsubsection{Product moments}

- By choosing $\mathcal{W}(t)$ as (2), (30) simplifies to

$$
\begin{aligned}
E\left(W_{1}^{n} W_{2}^{j}\right)= & (1-\rho)^{m_{2}} \sum_{k=0}^{\infty} \sum_{l=0}^{\infty} \frac{\left(m_{1}\right)_{k}\left(m_{2}-m_{1}\right)_{l} \rho^{k+l}}{l ! k !\left(m_{2}+k\right)_{l}} \frac{\Gamma\left(\frac{n}{\beta_{1}}+m_{1}+k\right) \Gamma\left(\frac{j+l}{\beta_{2}}+m_{2}+k\right)}{\Gamma\left(m_{1}+k\right) \Gamma\left(m_{2}+k\right)} \\
& \times\left(\frac{m_{1}}{\Omega_{1}(1-\rho)}\right)^{-\left(\frac{n}{\beta_{1}}\right)}\left(\frac{m_{2}}{\Omega_{2}(1-\rho)}\right)^{-\left(\frac{j+l}{\beta_{2}}-l\right)}
\end{aligned}
$$

for $n, j, m_{1}, m_{2}, \Omega_{1}, \Omega_{2}>0,-1<\rho<1$, and $m_{2} \geq m_{1}$.

- By choosing $\mathcal{W}(t)$ as (3) and using [8], p. 346, eq. 3.381.4, (30) simplifies to

$$
\begin{aligned}
E\left(W_{1}^{n} W_{2}^{j}\right)= & \frac{\left(\frac{v}{2}\right)^{v}}{\Gamma\left(\frac{v}{2}\right)}(1-\rho)^{m_{2}} \sum_{k=0}^{\infty} \sum_{l=0}^{\infty} \frac{\left(m_{1}\right)_{k}\left(m_{2}-m_{1}\right)_{l} \rho^{k+l}}{l ! k !\left(m_{2}+k\right)_{l}} \frac{\Gamma\left(\frac{n}{\beta_{1}}+m_{1}+k\right) \Gamma\left(\frac{j+l}{\beta_{2}}+m_{2}+k\right)}{\Gamma\left(m_{1}+k\right) \Gamma\left(m_{2}+k\right)} \\
& \times\left(\frac{m_{1}}{\Omega_{1}(1-\rho)} \frac{v}{2}\right)^{-\left(\frac{n}{\beta_{1}}\right)}\left(\frac{m_{2}}{\Omega_{2}(1-\rho)} \frac{v}{2}\right)^{-\left(\frac{j+l}{\beta_{2}}-l\right)} \Gamma\left(\frac{v}{2}-\left(\frac{j+l}{\beta_{2}}+\frac{n}{\beta_{1}}-l\right)\right) \quad \text { (49) }
\end{aligned}
$$

for $n, j, m_{1}, m_{2}, \Omega_{1}, \Omega_{2}, v>0,-1<\rho<1$, and $m_{2} \geq m_{1}$.

\subsection{4. $C d f$}

- By choosing $\mathcal{W}(t)$ as (2), (31) simplifies to

$$
\begin{aligned}
F_{\text {normal }}\left(w_{1}, w_{2}\right)= & (1-\rho)^{m_{2}} \sum_{k=0}^{\infty} \sum_{l=0}^{\infty} \frac{\left(m_{1}\right)_{k}\left(m_{2}-m_{1}\right)_{l}}{\left(m_{2}+k\right)_{l} k ! l !} \frac{\rho^{k+l}}{\Gamma\left(m_{1}+k\right) \Gamma\left(m_{2}+k\right)} \\
& \times \gamma\left(m_{1}+k, \frac{m_{1}}{\Omega_{1}(1-\rho)} w_{1}^{\beta_{1}}\right) \gamma\left(m_{2}+k+l, \frac{m_{2}}{\Omega_{2}(1-\rho)} w_{1}^{\beta_{2}}\right)
\end{aligned}
$$

for $m_{1}, m_{2}, \Omega_{1}, \Omega_{2}>0,-1<\rho<1$, and $m_{2} \geq m_{1}$.

- By choosing $\mathcal{W}(t)$ as (3), (31) simplifies to

$$
\begin{aligned}
F_{t}\left(w_{1}, w_{2}\right)= & \frac{\left(\frac{v}{2}\right)^{\frac{v}{2}}}{\Gamma\left(\frac{v}{2}\right)}(1-\rho)^{m_{2}} \sum_{k=0}^{\infty} \sum_{l=0}^{\infty} \frac{\left(m_{1}\right)_{k}\left(m_{2}-m_{1}\right)_{l}}{\left(m_{2}+k\right)_{l} k ! l !} \frac{\rho^{k+l}}{\Gamma\left(m_{1}+k\right) \Gamma\left(m_{2}+k\right)} \\
& \times \int_{0}^{\infty} \gamma\left(m_{1}+k, \frac{t m_{1}}{\Omega_{1}(1-\rho)} w_{1}^{\beta_{1}}\right) \gamma\left(m_{2}+k+l, \frac{t m_{2}}{\Omega_{2}(1-\rho)} w_{1}^{\beta_{2}}\right) t^{\frac{v}{2}-1} \exp \left(-\frac{v t}{2}\right) d t(51)
\end{aligned}
$$

for $m_{1}, m_{2}, \Omega_{1}, \Omega_{2}, v>0,-1<\rho<1$, and $m_{2} \geq m_{1}$. 


\section{REFERENCES}

1. N. Balakrishnan, and C. Lai, Bivariate probability distributions, $2^{\text {nd }}$ Edition. Springer-Verlag, New York. 2009.

2. A. Bekker, M. Arashi, and J.T. Ferreira, New bivariate gamma types with MIMO application, Communications in Statistics: Theory and Methods (https://doi.org/10.1080/03610926.2017.1417428). 2018.

3. S.H. Choi, P. Smith, B. Allen, W.Q. Malik, and M. Shafi, Severely fading MIMO channels: Models and mutual information, IEEE International Conference on Communications, pp. 4628-4633. 2007.

4. K. C. Chu, Estimation and decision for linear systems with elliptically random process, IEEE Transactions on Automatic Control, 18, pp. 499-505. 1973.

5. R.A.A. De Souza, and M.D. Yacoub, Bivariate Nakagami-m distribution with arbitrary correlation and fading parameters, IEEE Transactions on Wireless Communications, 7(12), pp. 5227-5232. 2008.

6. N.Y. Ermelova, and O. Tirkkonen, Cumulative distribution function of bivariate gamma distribution with arbitrary parameters and applications, IEEE Communications Letters, 19(2), pp. 167-170. 2015.

7. J.T. Ferreira, A. Bekker, and M. Arashi, Bivariate noncentral distributions: an approach via the compounding method, South African Statistical Journal, 50, pp. 103-122. 2016.

8. I.S. Gradshteyn, and I.M. Ryzhik, Table of Integral, Series, and Products, 7th Ed., Academic Press, Oxford, UK. 2007.

9. M. Kang, and M. Alouini, Capacity of correlated MIMO Rayleigh channels, IEEE Transactions on Wireless Communications, 5(1), pp. 143-155. 2006.

10. M. Kang, and M. Alouini, Capacity of MIMO Rician Channels, IEEE Transactions on Wireless Communications, 5(1), pp. 112-123. 2006.

11. J. Lopez-Fernandez, J.F. Paris, \& E. Martos-Naya, Bivariate Rician shadowed fading model, IEEE Transactions on Vehicular Technology, 67(1), pp. 378-384. 2018.

12. A.M. Mathai, A handbook of a generalised special functions for statistical and physical sciences, Oxford University Press, Oxford. 1993.

13. K.S. Miller, Complex stochastic processes: an introduction to theory and application, Addison-Wesley: New York. 1974.

14. M. Nakagami, The m-distribution - a general formula of intensity distribution of rapid fading, Statistical methods in radio wave propagation (Ed: Hoffman W.G.). Pergamon. 1960.

15. E. Ollila, J. Eriksson, and V. Koivunen, Complex elliptically symmetric random variables - generation, characterization, and circularity tests, IEEE Transactions on Signal Processing, 59(1), pp. 58-69. 2011.

16. P.B. Patnaik, The noncentral chi-square and F distributions and their applications. Biometrika, 36, pp. 202-232. 1949.

17. T. Piboongungon, V.A. Aalo,C.D. Iskander, and G.P. Efthymoglou, Bivariate generalised gamma distribution with arbitrary fading parameters, Electronics Letters, 41(12), pp. 709-710. 2005.

18. J. Reig, L. Rubio, and N. Cardona,Bivariate Nakagami-m distribution with arbitrary fading parameters, Electronics Letters, 38(25), pp. 1715-1717. 2002.

19. A. Saboor, S.B. Provost, and M. Ahmad, The moment generating function of a bivariate gamma-type distribution, Applied Mathematics and Computation, 218, pp. 11911-11921. 2012.

20. P.M. Shankar, Fading and shadowing in wireless systems, $2^{\text {nd }}$ edition. Springer-Verlag, New York. 2016.

21. M. Simon, and M. Alouini, Digital communication over fading channels. John Wiley and Sons, New York. 2005.

22. M.A.G. Villavicencio, RA.A. De Souza, G.C. de Souza, and M.D. Yacoub, A Bivariate $\kappa-\mu$ Distribution, IEEE Transactions on Vehicular Technology, 65(7), pp. 5737-5743. 2016.

23. J. Van den Berg, J.J.J. Roux, and A. Bekker, A Bivariate Generalization of Gamma Distribution. Communications in Statistics Theory and Methods, 42(19), pp. 3514-3527. 2013.

24. R.M. Yunus, and S. Khan, The bivariate noncentral chi-square distribution - a compound distribution approach. Applied Mathematics and Computation, 217, pp. 6237-6247. 2011.

25. R. Xu, Z. Zhong, J.M. Chen, and B. Ai, Bivariate gamma distribution from complex inverse Wishart matrix, IEEE Communications Letters, 13(2), pp. 118-120. 2009. 